\title{
The Analysis of the Properties of Bus Network Topology in Beijing Basing on Complex Networks
}

\author{
Hui Zhang, ${ }^{1}$ Peng Zhao, ${ }^{1}$ Jian Gao, ${ }^{1}$ and Xiang-ming Yao ${ }^{1,2}$ \\ ${ }^{1}$ School of Traffic and Transportation, Beijing Jiaotong University, Beijing 100044, China \\ ${ }^{2}$ State Key Laboratory of Rail Traffic Control and Safety, Beijing Jiaotong University, Beijing 100044, China \\ Correspondence should be addressed to Hui Zhang; zhanghui3052@gmail.com
}

Received 1 January 2013; Accepted 27 February 2013

Academic Editor: Yi-Kuei Lin

Copyright (c) 2013 Hui Zhang et al. This is an open access article distributed under the Creative Commons Attribution License, which permits unrestricted use, distribution, and reproduction in any medium, provided the original work is properly cited.

\begin{abstract}
The transport network structure plays a crucial role in transport dynamics. To better understand the property of the bus network in big city and reasonably configure the bus lines and transfers, this paper seeks to take the bus network of Beijing as an example and mainly use space $\mathrm{L}$ and space $\mathrm{P}$ to analyze the network topology properties. The approach is applied to all the bus lines in Beijing which includes 722 lines and 5421 bus station. In the first phase of the approach, space $\mathrm{L}$ is used. The results show that the bus network of Beijing is a scale-free network and the degree of more than 99 percent of nodes is lower than 10. The results also show that the network is an assortative network with 46 communities. In a second phase, space P is used to analyze the property of transfer. The results show that the average transfer time of Beijing bus network which is 1.88 and 99.8 percent of arbitrary two pair nodes is reachable within 4 transfers.
\end{abstract}

\section{Introduction}

Complex networks have been successfully used in many real complex systems since the researches of small-world networks and scale-free networks $[1,2]$. Many real complex systems have been well studied which include the actor network [1-3], WWW [2, 4], protein networks [5], and power grid $[1,3]$. During the last few years, transport networks, such as subway network [6], airport network [7-9], and street network [10], have been studied by the complex approach.

As an important part of urban transport systems and a trip mode to alleviate the traffic congestion, bus network has been studied by an increasingly large number of researchers. Sienkiewicz and Holyst studied the public transport in 22 Polish cities and found that the degree distribution of these network topologies followed a power law or an exponential function [11]. Xu et al. analyzed the three major cities of China, and they found that there is a linear behavior between strength and degree [12]. Soh et al. contribute a complex weighted network analysis of travel routes on the Singapore rail and bus transportation systems [13].

In this paper, we investigate the Beijing bus network (BBN) with 722 lines and 5421 nodes. The data can be achieved from the Internet (http://www.mapbar.com/ search/). In the network, the nodes stand for bus stations and edges (links) are the bus line connecting them along the route. In order to analyze the static properties of BBN, we use the so-called space $\mathrm{L}$ and space $\mathrm{P}$ to represent the $\mathrm{BBN}$. The space $\mathrm{L}$ is mainly used to analyze the properties of degree distribution, cluster, the average shortest path, degree correlation, and community structure. The space $\mathrm{P}$ is mainly used to analyze the average transfer time.

This paper is organized as follows. In Section 2, we give the description of the construction of the network. Section 3 analyzes the main properties of the BBN with space $\mathrm{L}$. In Section 4, we analyze the average transfer time of the BBN and use space $\mathrm{P}$ to calculate the smallest transfer times between any nodes in the network. Discussion and conclusions are given in Section 5.

\section{The Construction of the Network}

For easy calculation, there are some assumptions followed.

(1) The station name is the unique identification in the network. Do not account for the condition that some 


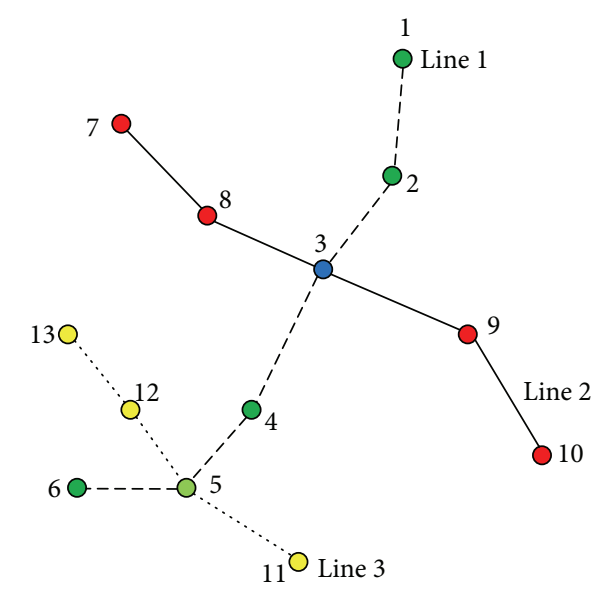

(a)

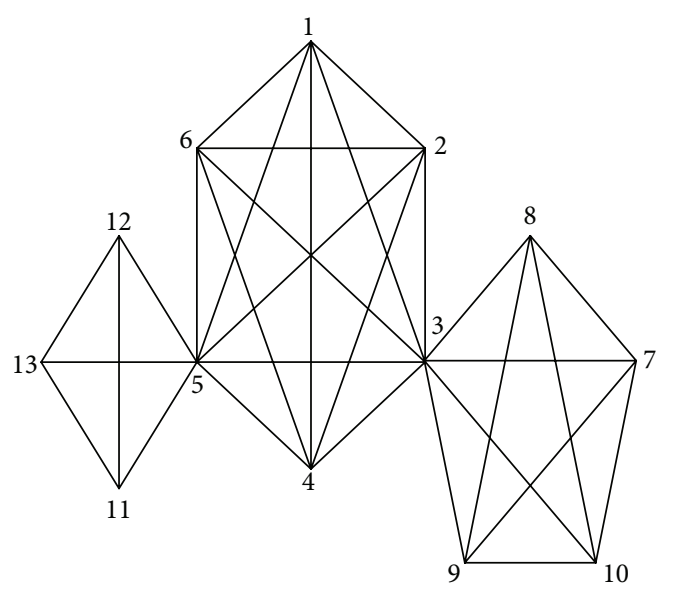

(b)

Figure 1: Illustration of space L (a) and space P (b).

stations have identical names but different parking place.

(2) The stations have slight difference between upstream line and downstream line. In this paper, the construction of the undirected network is based on the stations of the upstream lines.

(3) This paper does not consider the number of links between two stations and the frequency of bus; that is, it does not consider the weight of the network.

The bus network is usually represented by space L, space $P$ [11], space B [14], space C [15]. This paper will study and the Beijing bus network based on space $L$ and space P. Space $L$ consists of nodes which stand for the bus stations and a link between two nodes exists if they are consecutive stops on the route. Space $\mathrm{P}$ is a link formed between any two nodes of a line. Figure 1 gives a schematic representation of space $\mathrm{L}$ and space $P$.
TABLE 1: The stations of Beijing bus network with larger degree and the value of degree.

\begin{tabular}{lcc}
\hline Serial number & Bus station & degree \\
\hline 1 & Sanyuanqiao & 21 \\
2 & Liu Li Qiao Dong & 20 \\
3 & Beijing Xi station & 19 \\
4 & Liu Li Qiao Bei Li & 18 \\
5 & Beijing Zhangdong & 16 \\
6 & Madian Qiao Xi & 16 \\
7 & Xi Dao Kou & 16 \\
8 & Chong Wen Men Xi & 15 \\
9 & Guang An Men Nei & 14 \\
10 & Zuo Jia Zhuang & 14 \\
11 & Qianmen & 14 \\
12 & Dabei Yao Nan & 14 \\
13 & Tianqiao & 13 \\
14 & Ma Dian Qiao Nan & 13 \\
15 & Bei Tai Ping Qiao Xi & 13 \\
16 & Si Hui station & 13 \\
17 & Deshengmen & 13 \\
18 & Xi Bei Wang & 13 \\
19 & Xiyuan & 13 \\
20 & Xin Fa Di Qiao Bei & 13 \\
\hline
\end{tabular}

\section{The Main Properties of the Beijing Bus Network under Space $\mathbf{L}$}

Here we represent network as a graph $G=(V, E)$, where $V$ is the set of nodes and $E$ is the set of edges (links). $G$ is described by the $N \times N$ adjacency matrix $\left\{e_{i j}\right\}$. If there exists an edge between nodes $i$ and $j, e_{i j}=1$; otherwise $e_{i j}=0 . N$ is the number of nodes in the network. Figure 2 gives the Beijing bus network topology graph with 5421 nodes and 16986 links.

3.1. Degree. The degree $k_{i}$ of node $i$ is defined as the number of nodes that connected with the node $i$, which reflects the importance of the node $i$. In this paper, it refers to the number of bus stations with direct bus connecting with the current bus station. After calculation, the largest degree of the BBN is 21 , the smallest is 1 , and the average degree of all nodes is 3.13 which means one station averagely connects $3-4$ stations in Beijing bus network. Table 1 shows partly the bus stations of larger degree of BBN. 


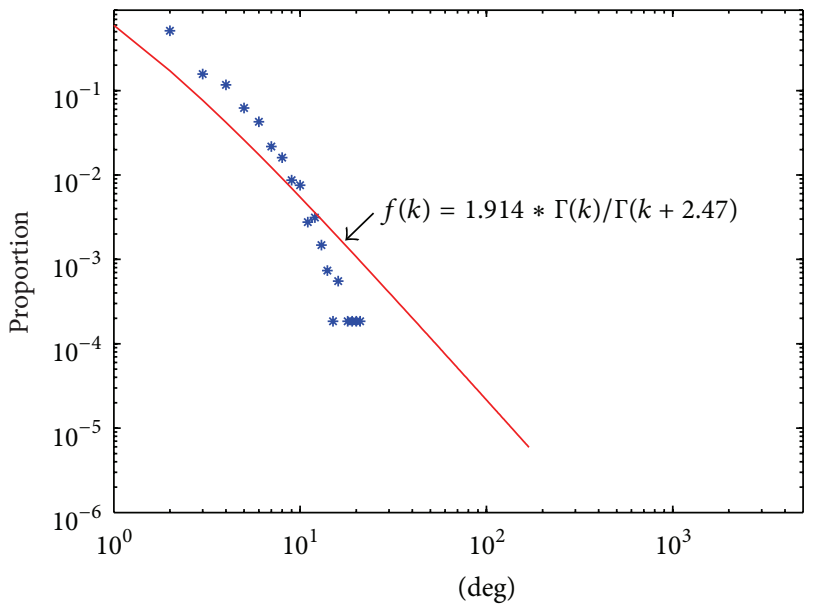

FIgURE 3: The degree distribution of Beijing bus network.

Here, we studied the proportion of the stations with the degree from 1 to 21 . Figure 3 shows the degree distribution of the stations in the BBN, and we found that it follows a shifted power law distribution $f(k)=1.914 * \Gamma(k) / \Gamma(k+2.47), k>$ $2, k$ is the degree, and the number of stations with degree 1 accounts for $4.48 \%$. It also shows that the degree of $99 \%$ of all station is smaller than 10 .

3.2. The Average Shortest Path. The average shortest path is the property to reflect the efficiency of information circulating on the network. It is defined as $l=2 * \sum_{i>j} d_{i j} /(N *$ $(N-1)), l$ is the average minimum shortest number of steps between all pairs of nodes, and $d_{i j}$ is the shortest path between node $i$ and node $j$.

3.3. Cluster Coefficient. Cluster coefficient is an important property of characterizing the local cohesiveness of the current node or the extent to which the nodes in the network are clustered together. In the BBN, clustering coefficient reflects the ease of the bus transport among the neighboring bus stations of the current one. It is defined as $C_{i}=A_{i} /\left(k_{i}\left(k_{i}-\right.\right.$ $1) / 2$ ), where $C_{i}$ is the cluster coefficient of node $i, A_{i}$ is the actual number of links between the neighbor nodes of the current node, and $k_{i}$ is the degree of node $i$. The cluster coefficient of the network is $C=\sum_{i \in G} C_{i} / N$.

3.4. Efficiency. The efficiency is the property to characterize the capacity of traffic, and it can be calculated with the formula $E=2 * \sum_{i>j}\left(1 / d_{i j}\right) /(N *(N-1))$, where $d_{i j}$ is the shortest path between node $i$ and node $j$ [16].

3.5. Degree Correlation. Degree correlation reflects the relationship between the degrees of nodes. Nodes with high degree tending to be connected with nodes with high degree are called assortativity. In contrast, nodes with high degree which have the tendency to be connected with low degree are
TABLE 2: The value of the main properties of Beijing bus network.

\begin{tabular}{lc}
\hline Network parameters & Value \\
\hline Number of nodes $(N)$ & 5421 \\
Number of links $(A)$ & 16986 \\
Number of lines $(L)$ & 722 \\
Average degree $(\mathrm{Ak})$ & 3.13 \\
Average shortest path $(l)$ & 20.03 \\
Cluster coefficient $(C)$ & 0.142 \\
Efficiency $(E)$ & 0.066 \\
Correlation coefficient $(r)$ & 0.185 \\
Number of communities $(Z)$ & 46 \\
Modularity $(Q)$ & 0.905 \\
\hline
\end{tabular}

called disassortativity. It can be calculated with the formula [17]

$$
r=\frac{M^{-1} \sum_{i} j_{i} k_{i}-\left[M^{-1} \sum_{i}(1 / 2)\left(j_{i}+k_{i}\right)\right]^{2}}{M^{-1} \sum_{i}(1 / 2)\left(j_{i}^{2}+k_{i}^{2}\right)-\left[M^{-1} \sum_{i}(1 / 2)\left(j_{i}+k_{i}\right)\right]^{2}},
$$

where $r$ is correlation coefficient and $j_{i}$ and $k_{i}$ are the degrees of the nodes at the ends of the $i$ th links, with $i=$ $1, \ldots, M$.

3.6. The Community Structure. It is usually found that there are many communities in one complex network; within the community, there are many links, but between the communities, there are fewer links [18]. Newman and Girvan [19] gave a measure $Q$ called modularity. For a division with $g$ communities, then define a $g \times g$ matrix $e$ whose component $e_{i j}$ is the fraction of edges in the original network that connects nodes in community $i$ to those in community $j$. The modularity is defined to be $Q=\sum_{i} e_{i i}-\sum_{i j k} e_{i j} e_{k i}=$ Tre $-\left\|e^{2}\right\|$, where $\|x\|$ indicates the sum of all elements of $x$. It can be achieved that $0 \leq Q \leq 1$. $Q=0$ indicates the community structure is not stronger than it would be expected by random chance. The larger the modularity is, the stronger the community structure is.

Table 2 shows the main properties of BBN. We can see that the average shortest path is 20.03 , which means people can reach destination by averagely taking 20 stops, and it is obvious that the BBN exhibits a small-world property. The cluster coefficient of $\mathrm{BBN}$ is 0.142 , which means the $\mathrm{BBN}$ is a sparse network. Furthermore, the correlated coefficient is 0.185 , which validates the result in the paper [m6] that when the number of nodes is larger than 500, the network is usually assortative. In addition, the community structure of BBN is so obvious and the modularity is 0.905 with 46 communities.

\section{The Transfer Property of Beijing Bus Network under Space $P$}

The transfer capacity is an important index to evaluate the performance of a bus network, and travelers always expect that they can reach the destination through the least number of transfers. In this paper, the average minimum transfer time 
TABLE 3: The specific network of Figure 1.

\begin{tabular}{cccccccccccccc}
\hline & $V_{1}$ & $V_{2}$ & $V_{3}$ & $V_{4}$ & $V_{5}$ & $V_{6}$ & $V_{7}$ & $V_{8}$ & $V_{9}$ & $V_{10}$ & $V_{11}$ & $V_{12}$ & $V_{13}$ \\
\hline$L_{1}$ & 1 & 1 & 1 & 1 & 1 & 1 & 0 & 0 & 0 & 0 & 0 & 0 & 0 \\
$L_{2}$ & 0 & 0 & 1 & 0 & 0 & 0 & 1 & 1 & 1 & 1 & 0 & 0 & 0 \\
$L_{3}$ & 0 & 0 & 0 & 0 & 1 & 0 & 0 & 0 & 0 & 0 & 1 & 1 & 1 \\
\hline
\end{tabular}

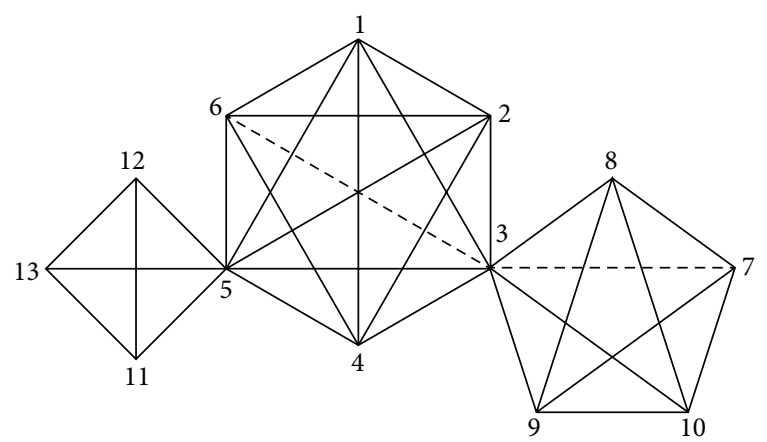

(a)

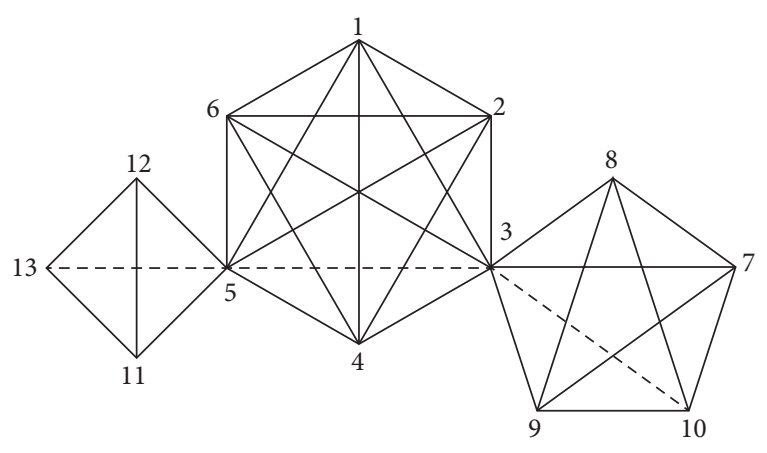

(b)

Figure 4: The illustration of transfer network under space P.

is used to evaluate the performance of the transfer capacity. Usually, travelers cannot reach the destination without transfer for a long distance trip, and the minimum transfer time between any two nodes is specific. The average minimum transfer time is the average among all pair nodes.

$G^{\prime}=(L, V)$ is used to represent the specific bus network, where $L$ is the line, $V$ is the bus station, and $G^{\prime}$ is a $L * N$ matrix $\left\{b_{i j}\right\}, b_{i j}=1$ if line $i$ stops at station $j$; otherwise $b_{i j}=$ 0 .

Table 3 shows the specific network given in Figure 1. We can achieve the minimum transfer time using Table 3. For example, (1) search $V_{6} \rightarrow V_{7}$. Because there is no directed line between the node $V_{6}$ and $V_{6}$, it needs transfer. From Table 3, we can see that $b_{16}=b_{13}=1$ through $L_{1}$, and $b_{23}=b_{27}=1$ through $L_{2}$, so we can achieve $V_{6} \stackrel{L_{1}}{\longrightarrow}$ $V_{3} \stackrel{L_{2}}{\longrightarrow} V_{7}$; that is, travelers need transfer at $V_{3}$ from $L_{1}$ to $L_{2}$. (2) Search $V_{10} \rightarrow V_{13}$. We can get the path $V_{10} \stackrel{L_{2}}{\longrightarrow} V_{3} \stackrel{L_{1}}{\longrightarrow}$ $V_{5} \stackrel{L_{3}}{\longrightarrow} V_{13}$ through two transfers.

Using the aforementioned method, we can get the minimum transfer time between any two nodes and calculate the
TABLE 4: The stations that have most line and the line numbers.

\begin{tabular}{|c|c|c|}
\hline Serial number & Station & Line numbers \\
\hline 1 & Sanyuanqiao & 47 \\
\hline 2 & Liu Li Qiao Bei Li & 40 \\
\hline 3 & Beijing Xi Zhan & 39 \\
\hline 4 & Zuo Jia Zhuang & 38 \\
\hline 5 & Liu Li Qiao Dong & 34 \\
\hline 6 & Liu Li Qiao Nan & 33 \\
\hline 7 & Dongzhimen Wai & 31 \\
\hline 8 & Gong Zhu Fen Nan & 31 \\
\hline 9 & Bei Da Di & 30 \\
\hline 10 & Bei Tai Ping Qiao Xi & 29 \\
\hline 11 & Jing An Zhuang & 29 \\
\hline 12 & Xiajia Hutong & 29 \\
\hline 13 & Qing $\mathrm{He}$ & 29 \\
\hline 14 & Xiyuan & 29 \\
\hline 15 & Beijing Zhangdong & 28 \\
\hline 16 & Xi Bei He & 28 \\
\hline 17 & Xiju & 28 \\
\hline 18 & Si Hui Zhan & 28 \\
\hline 19 & Liangmaqiao & 28 \\
\hline 20 & Mu xi yuan qiao dong & 27 \\
\hline 21 & Yan Huang yishu Guan & 26 \\
\hline 22 & Yuquanying Qiao Xi & 26 \\
\hline 23 & Dongwu Yuan & 25 \\
\hline 24 & Guang An Men Nei & 25 \\
\hline 25 & Qianmen & 25 \\
\hline 26 & Wanshou si & 25 \\
\hline 27 & Mu Xi Yuan Qiao Xi & 25 \\
\hline 28 & Kandan Qiao & 25 \\
\hline
\end{tabular}

average minimum transfer time. But it becomes very hard when the scale of network is becoming huge. In this paper, we use the space P to solve the problem. Firstly, we need to construct the network under space $P$, where the weight of the network is 1 . Secondly, the Floyd algorithm is used to achieve the shortest path between any two nodes. The shortest path value is the minimum line number that needs to use and the transfer time is the needed line number minus 1 . Figure 4 gives the illustration of the aforementioned example. Figure 4(a) shows $V_{6}$ can reach $V_{7}$ by using the two dotted lines. Figure 4(b) shows $V_{6}$ can reach $V_{7}$ by using the three dotted lines.

Here, we study the BBN. Table 4 shows the stations that have most lines. It is found that the station that owns most lines is San yuan qiao which has 47 lines.

Figure 5 shows the distribution of the proportion of the stations and the amount of line number; the result shows that it follow the exponent distribution $f(x)=0.79 * e^{-0.67 x}$, where $x$ is the number of lines. It is found that most stations of $\mathrm{BBN}$ own less than 10 lines and only 9 stations own more than 30 lines.

In this paper, the transfer time of BBN is studied by using space P. From Table 5, we can see that the most pair nodes 
TABLE 5: The proportion of the transfer time of Beijing bus network.

\begin{tabular}{lccccccc}
\hline atr & 0 & 1 & 2 & 3 & 4 & 4 more & unreachable \\
\hline Proportion & 0.0168 & 0.283 & 0.553 & 0.141 & 0.0047 & 0.00013 & 0.00137 \\
\hline
\end{tabular}

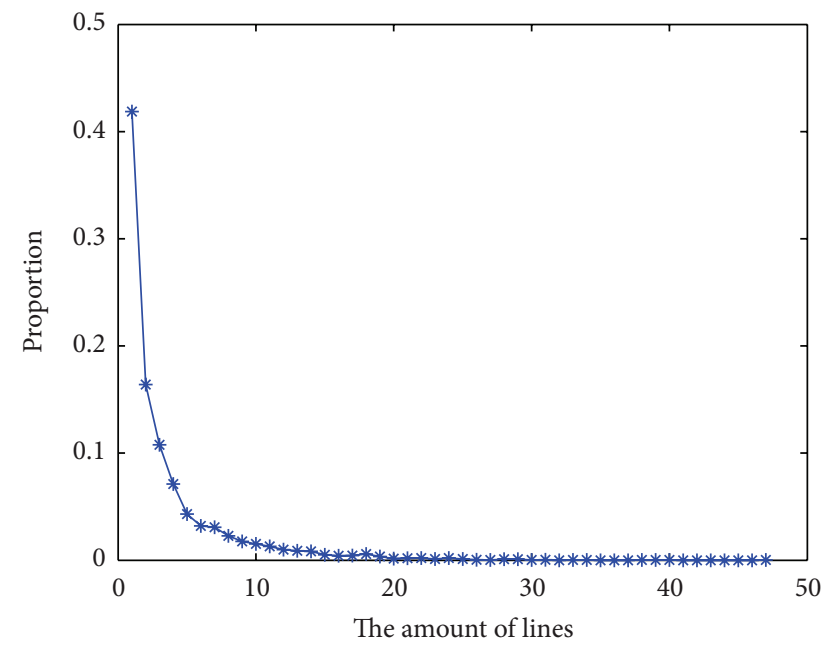

FIgURE 5: The distribution of a station's amount of lines.

are reachable through one or two transfers, and 99.85 percent of the pair nodes is reachable within four transfers and the average minimum transfer time atr $=1.88$. Usually, the larger the atr is, the worse the performance of the bus network is. In general, atr cannot be more than 2; otherwise, we can consider that the performance of the bus network is bad and travelers' trip is inconvenient. The transfer time of $\mathrm{BBN}$ is a little large and there is a room for improvement.

\section{Conclusion}

In this paper, space $\mathrm{L}$ and space $\mathrm{P}$ are used to analyze the static properties of Beijing bus network. Space L is used to research the main topology properties of the Beijing bus network. The results show the Beijing bus network has small cluster coefficient, scale-free feature, and assortative correlation and the community structure is obvious. Moreover, we research the transfer property using space $P$. The result shows that the accessibility of the Beijing bus network is good and the average minimum transfer time is 1.88 , which is a little large. A convenient bus network needs less transfers and high performance, and how to reduce transfer time and enhance the bus network dynamical performance is a valuable research.

\section{Acknowledgment}

This paper is supported by the Specialized Research Fund for the Doctoral Program of Higher Education of China (20120009110016).

\section{References}

[1] D. J. Watts and S. H. Strogatz, "Collective dynamics of "smallworld" networks," Nature, vol. 393, pp. 440-442, 1998.

[2] A.-L. Barabási and R. Albert, "Emergence of scaling in random networks," Science, vol. 286, no. 5439, pp. 509-512, 1999.

[3] E. Ravasz and A. L. Barabási, "Hierarchical organization in complex networks," Physical Review E, vol. 67, Article ID 026112, 7 pages, 2003.

[4] L. A. Adamic and B. A. Huverman, "Power-law distribution of the World Wide Web," Science, vol. 287, no. 5461, p. 2115, 2000.

[5] S. Maslov and K. Sneppen, "Specificity and stability in topology of protein networks," Science, vol. 296, no. 5569, pp. 910-913, 2002.

[6] V. Latora and M. Marchiori, "Is the Boston subway a smallworld network?” Physica A, vol. 314, no. 1-4, pp. 109-113, 2002.

[7] A. Barrat, M. Barthélemy, R. Pastor-Satorras, and A. Vespignani, "The architecture of complex weighted networks," Proceedings of the National Academy of Sciences of the United States of America, vol. 101, no. 11, pp. 3747-3752, 2004.

[8] R. Guimerà, S. Mossa, A. Turtschi, and L. A. N. Amaral, "The worldwide air transportation network: anomalous centrality, community structure, and cities' global roles," Proceedings of the National Academy of Sciences of the United States of America, vol. 102, no. 22, pp. 7794-7799, 2005.

[9] T. Jia and B. Jiang, "Building and analyzing the US airport network based on en-route location information," Physica A, vol. 391, no. 15, pp. 4031-4042, 2012.

[10] B. Jiang, "A topological pattern of urban street networks: universality and peculiarity," Physica A, vol. 384, no. 2, pp. 647655, 2007.

[11] J. Sienkiewicz and J. A. Holyst, "Statistical analysis of 22 public transport networks in Poland," Physical Review E, vol. 72, Article ID 046127, 11 pages, 2005.

[12] X. P. Xu, J. H. Hu, F. Liu, and L. S. Liu, "Scaling and correlations in three bus-transport networks of China," Physica A, vol. 374, no. 1, pp. 441-448, 2007.

[13] H. Soh, S. Lim, T. Zhang et al., "Weighted complex network analysis of travel routes on the Singapore public transportation system," Physica A, vol. 389, no. 24, pp. 5852-5863, 2010.

[14] K. A. Seaton and L. M. Hackett, "Stations, trains and smallworld networks," Physica A, vol. 339, no. 3-4, pp. 635-644, 2004.

[15] C. von Ferber, T. Holovatch, Y. Holovatch, and V. Palchykov, "Public transport networks: empirical analysis and modeling," The European Physical Journal B, vol. 68, no. 2, pp. 261-275, 2009.

[16] V. Latora and M. Marchiori, "Efficient behavior of small-world networks," Physical Review Letters, vol. 87, Article ID 198701, 4 pages, 2001.

[17] M. E. J. Newman, "Assortative mixing in networks," Physical Review E, vol. 89, Article ID 208701, 4 pages, 2002.

[18] M. E. J. Newman, "Detecting community structure in networks," The European Physical Journal B, vol. 38, no. 2, pp. 321330, 2004. 
[19] M. E. J. Newman and M. Girvan, "Finding and evaluating community structure in networks," Physical Review E, vol. 69, Article ID 026113, 15 pages, 2004. 


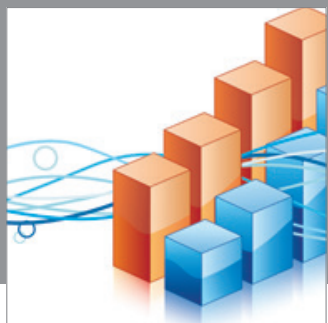

Advances in

Operations Research

mansans

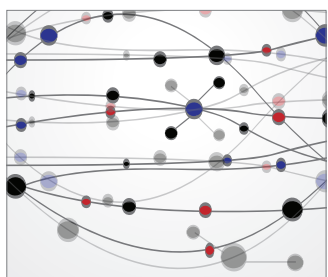

The Scientific World Journal
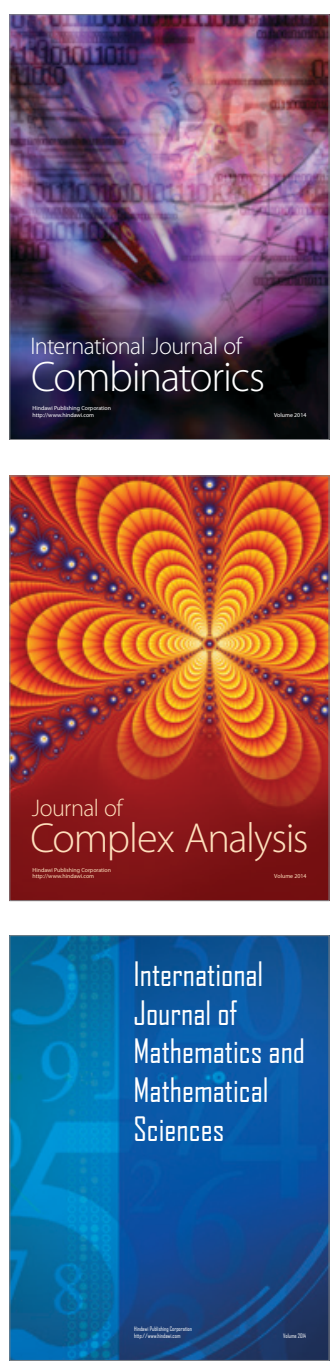
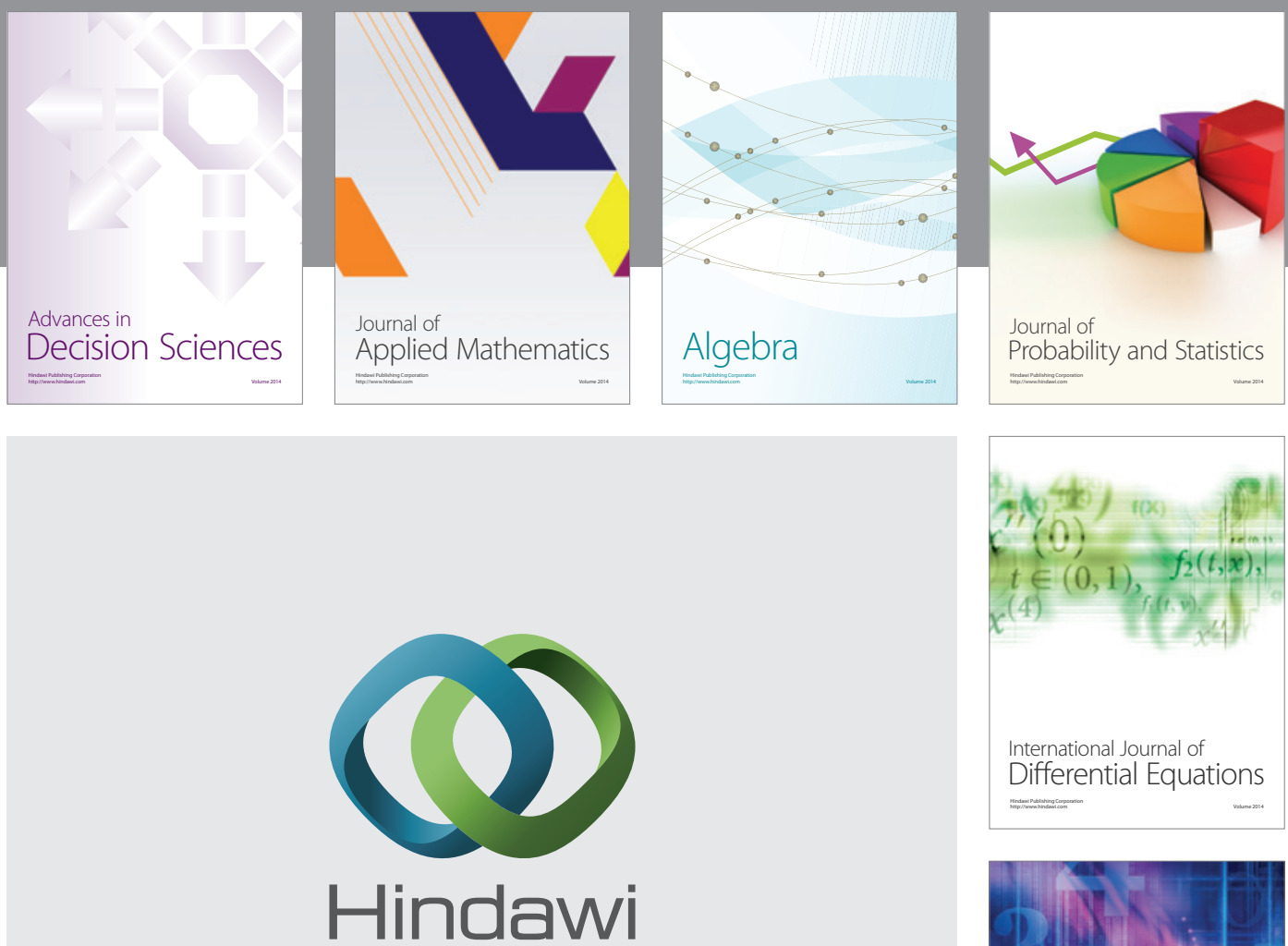

Submit your manuscripts at http://www.hindawi.com
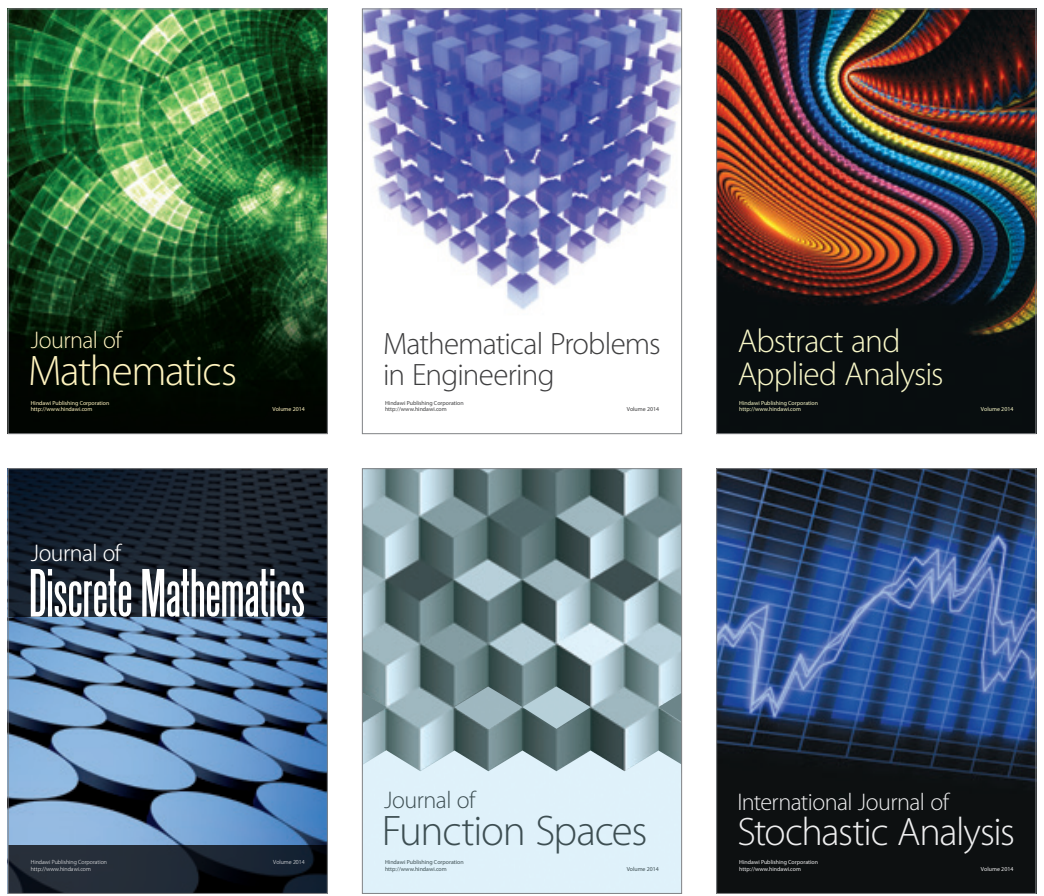

Journal of

Function Spaces

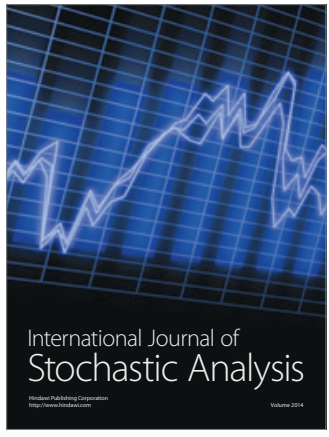

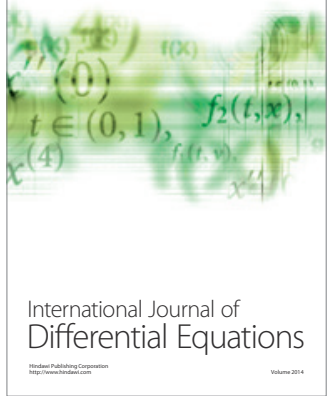
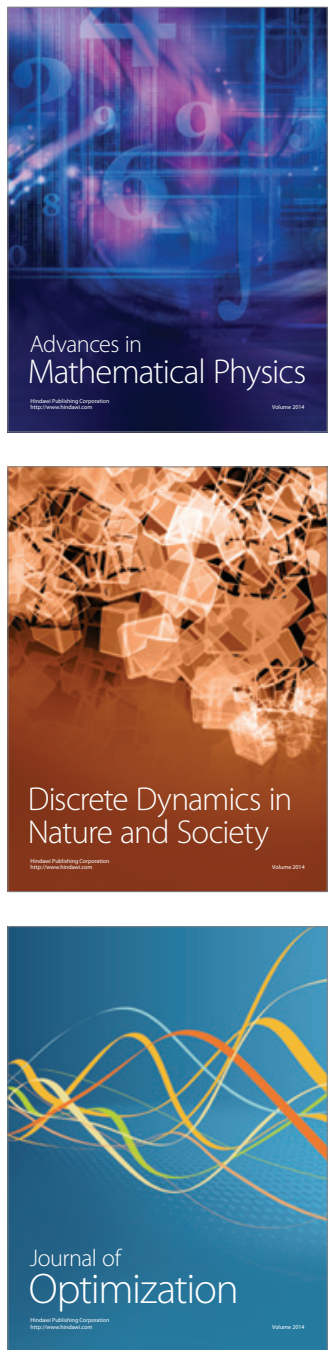